\title{
I manoscritti secretati di Giovanni Verga
}

\section{Gabriella Alfieri}

PUBBLICATO: 27 MARZO 2021

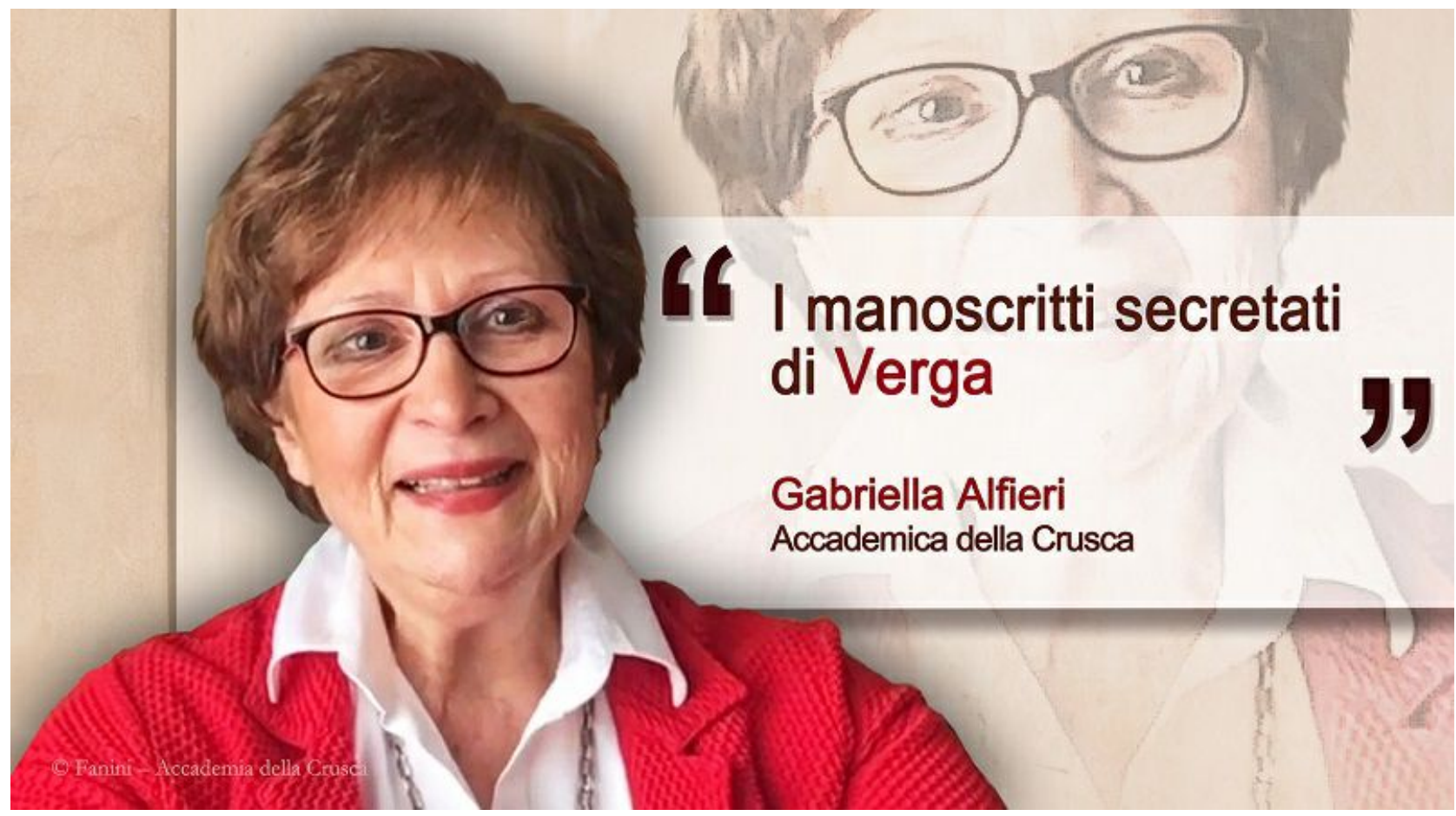

$\mathrm{D}$ all'osservatorio privilegiato della Fondazione Verga e del Comitato per l'Edizione Nazionale dello scrittore, vorrei riproporre un caso storico-critico e filologico, protrattosi per novant'anni, e riguardante un autore che, per il rivoluzionario italiano etnificato dei Malavoglia e di Vita dei campi, Bruno Migliorini aveva inserito nella sua Cronologia della lingua italiana (1975). I manoscritti di Giovanni Verga, a lungo secretati, poi per metà riscattati e per metà creduti dispersi ma fortunosamente recuperati, costituiscono infatti un caso tuttora per certi aspetti aperto.

Tutto ebbe inizio nel gennaio I922 quando lo scrittore ottantaduenne si spegneva a Catania, mentre era intento a catalogare con acribia filologica gli autografi delle proprie opere narrative e teatrali. Con la parsimonia tipica di quelle generazioni, da alcuni anni andava riponendo i suoi manoscritti nelle cartelline di cartone con l'intestazione "Senato del Regno", gratuitamente disponibili dopo la sua nomina a senatore a vita nel I920. Nel caso di stesure plurime di un'opera Verga identificava ogni redazione con il titolo provvisorio, aggiungendo tra parentesi quello definitivo in unideale seriazione delle varianti. Cosi le tre versioni del romanzo Eros sono tuttora conservate nel Fondo Verga della Biblioteca Regionale Universitaria di Catania in tre cartelline senatoriali, etichettate Aporeo (Eros). Per l'intera casistica si rinvia a Lo scrittoio del verista di Francesco Branciforti (in I tempi e le opere di Giovanni Verga, Firenze, Le Monnier 1987).

Ad affiancarlo nell'oneroso lavoro, che - se compiuto - sarebbe risultato di capitale importanza per i futuri studiosi, Verga aveva chiamato l'amico e sodale Federico De Roberto, che, dopo la morte del Maestro, prosegui l'archiviazione con amorevole cura e con analogo rigore filologico. Purtroppo però anche De Roberto mori improvvisamente nel I927, lasciando incompleta la sua ricognizione storico- 
critica.

Al contrario delle carte manzoniane, provvidenzialmente rimaste sotto il tetto natio e, grazie alle cure dei piu autorevoli filologi italiani passate definitivamente alla Biblioteca Nazionale di Brera, i manoscritti e le lettere dell'autore dei Malavoglia avrebbero affrontato unodissea, passando dall'amorevole e competente cura di De Roberto alle possessive e dilettantesche attenzioni di filologi improvvisati.

Inizia da qui la fortunosa vicenda dei manoscritti verghiani: il nipote ed erede Giovannino Verga Patriarca, fin troppo accogliente verso gli estimatori dello zio, apriva la casa di Via Sant'Anna a tutti i potenziali studiosi, senza verificarne la qualificazione e gli intenti. Fu cosi che, grazie all'intervento del Ministro Giuseppe Bottai, di cui erano amici, i fratelli Lina e Vito Perroni ottennero 'in prestito' tutti i manoscritti verghiani, col mandato di pubblicare l'Opera Omnia dello scrittore. Dopo la rinuncia di Bemporad, il contratto fu stipulato da Mondadori. Dalla stazione ferroviaria di Catania partivano cosi nel I929, con destinazione il domicilio romano dei Perroni, parecchie casse in cui erano stipate tutte le carte verghiane.

Nonostante i ripetuti annunci, i Perroni non editarono nessuna opera, limitandosi a pubblicare stralci di testi narrativi ed epistolari i cui autografi erano di immediata lettura e il cui interesse era più aneddotico che filologico-critico: frammenti del romanzo giovanile Amore e Patria; lettere confidenziali alla madre; schematici abbozzi dei capolavori. Loperazione editoriale presentata come Opera Omnia consisteva in stampe "emendate" sull'autografo o sulle edizioni originali, con l'introduzione di errori ascrivibili a maldestra lettura.

Come testimonia il fitto carteggio tra Mondadori, Giovannino Verga e i Perroni che attraversa il trentennio I940-I970, l'editore consigliò ripetutamente all'erede di Verga di farsi restituire i manoscritti, sperando di poter affidare l'edizione a curatori più capaci e affidabili. Non ottenendo risultati, finanziò la microfilmatura del prezioso corpus. A tutt'oggi il "Fondo Mondadori" è l'unica testimonianza organica degli autografi verghiani prima dell'improvvido smembramento di cui diremo.

Intanto si era mobilitato il mondo delle istituzioni. Nel 1957 l'onorevole catanese Matteo Gaudioso promosse un'interrogazione parlamentare sollecitando il Ministero dell'Istruzione a recuperare un bene culturale cosi prezioso. Si provvide presso casa Perroni alla prima "notifica di notevole interesse", che avrebbe dovuto preludere a interventi risolutivi delle autorità. Le notifiche si replicarono fino al I973, ma gli elenchi riportano sempre gli stessi titoli, che non rispecchiano la consistenza degli autografi testimoniata dal Fondo di microfilm Mondadori. Effettivamente le istituzioni sembravano anestetizzate rispetto a una questione filologico-critica di capitale importanza per la cultura nazionale, che avrebbe richiesto interventi energici e radicali. Per una serie di ragioni difficili oggi da ricostruire, nessun intervento risolutivo si ebbe da parte delle autorità.

Già dagli anni Sessanta si era levata, trovando ampia eco nella stampa nazionale, la protesta di intellettuali sulla "cattività" dei manoscritti verghiani. Poeti come Montale e Quasimodo, scrittori come Bonaviri e critici come Luigi Russo, biasimarono Vito Perroni (la sorella aveva rinunciato all'incarico) perché non solo non produceva nessuna edizione critica delle opere verghiane, come aveva promesso da decenni, ma soprattutto impediva la libera consultazione dei manoscritti, continuando a detenerli abusivamente. Nella campagna di stampa intervennero anche arguti vignettisti, che raffiguravano la vicenda in chiave di rapina o estorsione, ma non si ebbero effetti di sorta.

Fu invece la famiglia Verga a sbloccare la situazione, ottenendo, dopo una battaglia legale col Perroni, la restituzione dei manoscritti. Nel 1975 il Tribunale di Catania attribui a Pietro Verga, figlio ed erede 
di Giovannino, la proprietà legale di tutti i manoscritti, compresi quelli non notificati, ingiungendone la restituzione. Nel corso del 1977 effettivamente il Perroni consegnò all'erede Verga dei manoscritti, che costituiscono il Fondo Verga acquistato dalla Regione Siciliana nell'ottobre del 1977 e attualmente conservato presso la Biblioteca Regionale Universitaria di Catania. Ma tratteneva presso di sé una cospicua parte di autografi, avanzando la necessità di un più adeguato studio descrittivo. Si concludeva così, seppur con un recupero lacunoso, il mezzo secolo di "cattività" delle carte verghiane, e si avviava finalmente l'Edizione Nazionale, affidata a veri e accreditati filologi. Tuttavia all'appello mancavano quasi metà delle carte e un consistente numero di lettere tuttora disperse ma sicuramente sopravvissute, come testimonia la periodica ricomparsa di gruppi più o meno consistenti di carteggi nel mercato antiquario. Nel 2012 sono stati acquisiti dalla Regione Siciliana nuclei importanti di lettere di Verga ai principali intellettuali contemporanei come Giacosa e alla famiglia. Nel 2016 un autografo di Cavalleria rusticana e un cospicuo numero di lettere verghiane emergeva a Parigi in una vendita all'asta poi precipitosamente annullata.

Ma il vero colpo di scena riguardava i manoscritti dispersi dal 1977. Nel febbraio 2013 una nota casa d'aste metteva in vendita a Milano un cospicuo corpus di autografi e lettere di Giovanni Verga, presentato come "Fondo Vito Perroni", che comprendeva opere letterarie, teatrali e documenti di varia natura e di vario interesse, insieme a volumi della Biblioteca di Verga. Il convergente impegno di enti culturali e scientifici (Fondazione Verga e Comitato per l'Edizione Nazionale delle Opere di Giovanni Verga), di istituzioni nazionali e regionali (Direzione generale per le biblioteche, gli istituti culturali e il diritto d'autore del MIBAC; Soprintendenza Archivistica e Bibliografica della Lombardia; Soprintendenza ai Beni archivistici e librari del Lazio) e del Nucleo dei Carabinieri per la Tutela del Patrimonio Culturale, avrebbe portato al tempestivo recupero del prezioso corpus, attivando le normali procedure di legge per evitarne la vendita impropria. Il fondo, ridenominato "Fondo ex Vito Perroni”, veniva sottoposto a notifica e poi per ordine dell'autorità giudiziaria a sequestro cautelativo presso il Centro per la Tradizione manoscritta fondato da Maria Corti all'Università di Pavia, dove, su iniziativa di Carla Riccardi e delle Presidenti via via avvicendatesi (Maria Antonietta Grignani, Clelia Martignoni, Gianfranca Lavezzi), i manoscritti sono stati restaurati e conservati in adeguate condizioni di protezione e sicurezza. Nel 2016 il Comitato per l'Edizione Nazionale delle Opere di Giovanni Verga ne ha predisposto, con l'autorizzazione della magistratura, la riproduzione digitale, in modo che i manoscritti attualmente sequestrati vengano preservati, indipendentemente dall'assegnazione agli aventi diritto, tuttora da individuare. Sono stati digitalizzati anche i microfilm Mondadori, ricomponendo idealmente, seppur in riproduzione, un corpus che costituisce un inestimabile bene culturale per l'Italia e non solo.

Nell'attesa che si definisca giuridicamente e giudiziariamente la vicenda, il risultato fondamentale è l'insperato recupero di un patrimonio testuale sopravvissuto solo nella provvidenziale riproduzione dei microfilm Mondadori, in cui tuttavia mancano cospicui nuclei di lettere e i manoscritti di romanzi giovanili come Amore e Patria, che figurano nella nostra riproduzione. La complementarità delle riproduzioni sembra prefigurare la complementarità tra il Fondo Verga della Biblioteca Regionale Universitaria di Catania e il cosi detto "Fondo ex Vito Perroni", facendo desiderare che la "paziente intraprendenza" auspicata da Francesco Branciforti nel I987, quando inaugurava l'Edizione Nazionale verghiana, possa spingere le autorità preposte alla tutela dei beni culturali, ad assumere le iniziative piu idonee per la definitiva soluzione del caso. Il centenario della morte dello scrittore, che cade l'anno prossimo, potrebbe essere una bella occasione per liberare le carte verghiane da una secretazione quasi secolare. 


\section{Cita come:}

Gabriella Alfieri, I manoscritti secretati di Giovanni Verga , "Italiano digitale", 2021, XVI, 2021/1 (gennaio-marzo)

DOI: $10.35948 / 2532-9006 / 2021.6498$

Copyright 2021 Accademia della Crusca

Pubblicato con licenza creative commons CC BY-NC-ND 